\title{
Exact and approximate solutions for the fractional Schrödinger equation with variable coefficients
}

\author{
Baojian Hong ${ }^{1 *}$ (D), Dianchen Lu² and Wei Chen ${ }^{3}$
}

\footnotetext{
*Correspondence: hbj@njit.edu.cn ${ }^{1}$ Department of Mathematical Physics, Nanjing Institute of Technology, Nanjing, P.R. China Full list of author information is available at the end of the article
}

\begin{abstract}
In this paper, by introducing the fractional derivatives in the sense of Caputo, the modified general mapping deformation method (MGMDM) and the modified fractional variational iteration method (MFVIM) are applied to obtain some exact and approximate solutions of the variable-coefficient fractional Schrödinger equation (VFNLS) with time and space fractional derivatives. With the aid of symbolic computation, a broad class of exact analytical solutions and their structure of the VFNLS are investigated. Furthermore, the approximate iterative series showed that the MFVIM is powerful, reliable and effective when compared with some traditional decomposition method in searching for the approximate solutions of the complex nonlinear partial differential equations with variable coefficients and fractional derivatives.

Keywords: Modified fractional variational iteration method; Caputo derivative; Fractional nonlinear Schrödinger equation; Exact solutions; Approximate solutions
\end{abstract}

\section{Introduction}

In recent years, due to the wide applications of fractional differential equations (FDEs) in nonlinear science [1], many phenomena can be described successfully by using FDEs such as chaotic oscillations [2], electrochemistry [3], engineering [4] and so on [5]. Searching for exact solutions of these FDEs plays an important and significant role in the study on the dynamics of those phenomena. Many powerful methods have been proposed to handle this subject, such as the Darboux transformation method [6], the split-step Fourier transform method [7], the fractional characteristic method [8,9]. But because of the complexity of the nonlinear terms, most FDEs do not have exact analytic solutions, so approximate and numerical methods must be used. Many efforts have been proposed for these problems, including the homotopy analysis method (HAM) [10], the homotopy perturbation method (HPM) [11], the adomian decomposition method (ADM) [12].

The variational iteration method (VIM) and the fractional variational iteration method (FVIM) were established in [13] and [14, 15] by He, respectively, and they were thoroughly used by many researchers [16, 17]. Some new developments about the fractional derivative and its application are available in Refs. [18-20]. After giving some modification, Hong and Lu proposed the MFVIM for some complex nonlinear partial differential equations

(c) The Author(s) 2019. This article is distributed under the terms of the Creative Commons Attribution 4.0 International License (http://creativecommons.org/licenses/by/4.0/), which permits unrestricted use, distribution, and reproduction in any medium, provided you give appropriate credit to the original author(s) and the source, provide a link to the Creative Commons license, and indicate if changes were made. 
with fractional derivative [21]. The motivation of this paper is to construct some exact and approximate solutions for the VFNLS by using the MGMDM and MFVIM.

We give some definitions and properties of the fractional calculus theory which are used further in this paper [22-24].

Definition 1 The fractional derivative is defined as the following limit form:

$$
f^{\alpha}=\lim _{h \rightarrow 0} \frac{\Delta^{\alpha}[f(x)-f(0)]}{h^{\alpha}} .
$$

Definition 2 The Riemann-Liouville fractional integral operator of order $\alpha>0$ for a function $f(x)$ is defined as

$$
J_{x}^{\alpha} f(x)=\frac{1}{\Gamma(\alpha)} \int_{0}^{x}(x-\xi)^{\alpha-1} f(\xi) d \xi, \quad \alpha>0, x>0, J_{x}^{0} f(x)=f(x) .
$$

Also we have the following properties:

$$
J^{\alpha} J^{\beta} f(x)=J^{\alpha+\beta} f(x), \quad J^{\alpha} J^{\beta} f(x)=J^{\beta} J^{\alpha} f(x), \quad J^{\alpha} x^{\gamma}=\frac{\Gamma(\gamma+1)}{\Gamma(\alpha+\gamma+1)} x^{\alpha+\gamma} .
$$

Definition 3 For $\alpha>0, x>0, f(x) \in C_{-1}^{n}$, the Caputo fractional derivative operator of order $\alpha$ on the whole space is defined as

$$
D^{\alpha} f(x)=J^{n-\alpha} D^{n} f(x)= \begin{cases}\frac{1}{\Gamma(n-\alpha)} \int_{0}^{x}(x-\xi)^{n-\alpha-1} f^{(n)}(\xi) d \xi, & n-1<\alpha<n, n \in N . \\ \frac{d^{(n)} f(x)}{d x^{n}}, & \alpha=n .\end{cases}
$$

We have the following properties:

$$
\begin{aligned}
& D^{\alpha} C=0 \quad(C \text { is a constant }), \quad D^{\alpha} x^{\gamma}=\left\{\begin{array}{l}
\frac{\Gamma(\gamma+1)}{\Gamma(\gamma-\alpha+1)} x^{\gamma-\alpha}, \quad \gamma>\alpha-1, \\
0, \quad \gamma \leq \alpha-1 .
\end{array}\right. \\
& J^{\alpha} D^{\alpha} f(x)=f(x)-\sum_{k=0}^{n-1} f^{(k)}\left(0^{+}\right) \frac{x^{k}}{k !}, \quad n-1<\alpha<n, \quad D^{\alpha} J^{\alpha} f(x)=f(x) .
\end{aligned}
$$

Definition 4 The fractional derivative of compounded functions is defined as

$$
d^{\alpha} f=\Gamma(1+\alpha) f .
$$

Definition 5 The integral with respect to $d(x)^{\alpha}$ is defined as the solution of the fractional differential equation

$$
d y=f(x) d(x)^{\alpha}, \quad x \geq 0, y(0)=0 .
$$

\section{Exact solution and their structure of the GFNLS}

Consider the following generalized time and space fractional nonlinear Schrödinger equation with variable coefficients:

$$
i \frac{\partial^{\alpha} u}{\partial z^{\alpha}}+\frac{1}{2} a(z) \frac{\partial^{2 \beta} u}{\partial t^{2 \beta}}+b(z) u|u|^{2}-i c(z) u=0, \quad z>0,0<\alpha, \beta \leq 1,
$$


where $u=u(z, t), \frac{\partial u^{\alpha}}{\partial z}=D_{z}^{\alpha} u, \frac{\partial^{2 \beta} u}{\partial t^{2 \beta}}=D_{t}^{\beta}\left(D_{t}^{\beta} u\right)$, when $\alpha=\beta=1$, this equation turns to the famous nonlinear Schrödinger equations in an optical fiber [25-27]. Here $u(z, t)$ is the complex envelope of the electrical field, $z$ is the normalized propagation distance along the fiber, $t$ is the retarded time and the subscripts denote partial derivatives, the real analytic functions $a(z)$ and $b(z)$ are the slowly increasing dispersion coefficient and nonlinear coefficient, respectively, which represent the group velocity dispersion (GVD) and the self-phase modulation (SPM), $c(z)$ represents the heat-insulating amplification or loss. The transmission of a soliton in the real communication system of an optical soliton is described by Eq. (1) [9].

If we let $t \rightarrow x, z \rightarrow t$, Eq. (1) turns to the following form:

$$
i \frac{\partial^{\alpha} u}{\partial t^{\alpha}}+\frac{1}{2} a(t) \frac{\partial^{2 \beta} u}{\partial x^{2 \beta}}+b(t) u|u|^{2}-i c(t) u=0, \quad t>0,0<\alpha, \beta \leq 1 .
$$

We can give the complex variable transformation as follows:

$$
\begin{aligned}
& u=A(t) \varphi(\xi) e^{i \eta} \\
& \xi=\frac{k_{1} x^{\beta}}{\Gamma(1+\beta)}+\frac{1}{\Gamma(\alpha)} \int_{0}^{t}(t-\tau)^{\alpha-1} c_{1}(\tau) d \tau, \\
& \eta=\frac{k_{2} x^{\beta}}{\Gamma(1+\beta)}+\frac{1}{\Gamma(\alpha)} \int_{0}^{t}(t-\tau)^{\alpha-1} c_{2}(\tau) d \tau,
\end{aligned}
$$

with the following consistency conditions:

$$
A(t)=k e^{\frac{\alpha}{\Gamma(1+\alpha)} \int_{0}^{t} \tau^{\alpha-1} c(\tau) d \tau}, \quad c_{1}(t)=-k_{1} k_{2} a(t),
$$

where $k, k_{1}, k_{2}$ are arbitrary nonzero constants.

Substituting (3), (4), (5) into (2), we obtain

$$
k_{1}^{2} a(t) \varphi_{\xi \xi}(\xi)-\left[2 c_{2}(t)+k_{2}^{2} a(t)\right] \varphi(\xi)+2 b(t) A^{2}(t) \varphi^{3}(\xi)=0,
$$

where $\varphi_{\xi \xi}(\xi)=\frac{d^{2} \varphi(\xi)}{d \xi^{2}}$, with the idea of the homogeneous balance principle, by balancing the highest-order linear term $\varphi_{\xi \xi}(\xi)$ and the nonlinear $\varphi^{3}(\xi)$ in (6), we assume that Eq. (6) has the following solutions:

$$
\begin{aligned}
& \varphi=\varphi(\xi)=A_{0}+A_{1} F(\xi)=A_{0}+A_{1} F, \\
& F^{\prime 2}=\sum_{i=0}^{4} a_{i} F^{i},
\end{aligned}
$$

where $a_{i}(i=0,1,2,3,4)$ are constants to be determined. We write $F=F(\xi)$ and $F^{\prime}=\frac{d F(\xi)}{d \xi}$. Substituting (7) and (8) into (6), and setting the coefficients of $F^{i}(\xi), i=0,1,2, \ldots$, to zero yield an ODE with respect to the unknowns $A_{0}, A_{1}, a_{0}, a_{1}, a_{2}, a_{3}, a_{4}, k_{1}, k_{2}, a(t), b(t)$ and $c_{2}(t)$. After solving the ODE by Mathematica software we could determine the following solutions: 
Case 1

$$
\begin{aligned}
& A_{0}=a_{1}=a_{3}=0, \quad A_{1}=\mathrm{const}, \quad c_{2}(t)=\frac{1}{2}\left(a_{2} k_{1}^{2}-k_{2}^{2}\right) a(t), \\
& b(t)=-a_{4} k_{1}^{2} A_{1}^{-2} a(t) A^{-2}(t) .
\end{aligned}
$$

By using the general mapping deformation method [28], we can obtain the following solutions of the corresponding Eq. (2):

$$
\begin{aligned}
& u_{1_{j}}=k A_{1} e^{\frac{\alpha}{\Gamma(1+\alpha)} \int_{0}^{t} \tau^{\alpha-1} c(\tau) d \tau+i\left[\frac{k_{2} x^{\beta}}{\Gamma(1+\beta)}+\frac{1}{2 \Gamma(\alpha)} \int_{0}^{t}(t-\tau)^{\alpha-1}\left(a_{2} k_{1}^{2}-k_{2}^{2}\right) a(\tau) d \tau\right]} F_{1_{j}}\left(\xi_{1_{j}}\right), \\
& \xi_{1_{j}}=\frac{k_{1} x^{\beta}}{\Gamma(1+\beta)}-\frac{k_{1} k_{2}}{\Gamma(\alpha)} \int_{0}^{t}(t-\tau)^{\alpha-1} a(\tau) d \tau, \quad j=1,2,3, \ldots
\end{aligned}
$$

We have the consistency conditions

$$
b(t)=-a_{4} k_{1}^{2} A_{1}^{-2} a(t) A^{-2}(t) .
$$

Here $F_{1_{j}}$ is an arbitrary solution of the equation $F_{1_{j}}^{\prime 2}=a_{0_{j}}+a_{2_{j}} F_{1_{j}}^{2}+a_{4_{j}} F_{1_{j}}^{4}$,we can obtain the 52 classes of exact solutions $F_{1_{j}}$ from Ref. [29]; for example, if we let $a_{0_{1}}=1-m^{2}$, $a_{2_{1}}=2 m^{2}-1, a_{4_{1}}=-m^{2}, F_{1_{1}}=c n \xi$, we have

$$
u_{1_{1}}=k A_{1} e^{\frac{\alpha}{\Gamma(1+\alpha)} \int_{0}^{t} \tau^{\alpha-1} c(\tau) d \tau+i\left[\frac{k_{2} x^{\beta}}{\Gamma(1+\beta)}+\frac{1}{2 \Gamma(\alpha)} \int_{0}^{t}(t-\tau)^{\alpha-1}\left(\left(2 m^{2}-1\right) k_{1}^{2}-k_{2}^{2}\right) a(\tau) d \tau\right]} \operatorname{cn}\left(\xi_{1_{j}}\right),
$$

with the consistency conditions $b(t)=m^{2} k_{1}^{2} A_{1}^{-2} a(t) A^{-2}(t), A(t)=k e^{\frac{\alpha}{\Gamma(1+\alpha)} \int_{0}^{t} \tau^{\alpha-1} c(\tau) d \tau}$, if we let $a_{0_{2}}=1, a_{2_{2}}=-1-m^{2}, a_{4_{2}}=m^{2}, F_{1_{2}}=s n \xi$, we have

$$
u_{1_{2}}=k A_{1} e^{\frac{\alpha}{\Gamma(1+\alpha)} \int_{0}^{t} \tau^{\alpha-1} c(\tau) d \tau+i\left[\frac{k_{2} x^{\beta}}{\Gamma(1+\beta)}+\frac{1}{2 \Gamma(\alpha)} \int_{0}^{t}(t-\tau)^{\alpha-1}\left(\left(-m^{2}-1\right) k_{1}^{2}-k_{2}^{2}\right) a(\tau) d \tau\right]} \operatorname{sn}\left(\xi_{1_{j}}\right),
$$

with the consistency conditions $b(t)=-m^{2} k_{1}^{2} A_{1}^{-2} a(t) A^{-2}(t)$, if we let $a_{0_{3}}=m^{2}-1, a_{2_{3}}=$ $2-m^{2}, a_{4_{3}}=-1, F_{1_{3}}=d n \xi$, we derive

$$
u_{1_{3}}=k A_{1} e^{\frac{\alpha}{\Gamma(1+\alpha)} \int_{0}^{t} \tau^{\alpha-1} c(\tau) d \tau+i\left[\frac{k_{2} x^{\beta}}{\Gamma(1+\beta)}+\frac{1}{2 \Gamma(\alpha)} \int_{0}^{t}(t-\tau)^{\alpha-1}\left(\left(2-m^{2}\right) k_{1}^{2}-k_{2}^{2}\right) a(\tau) d \tau\right]} d n\left(\xi_{1_{j}}\right),
$$

with the consistency conditions $b(t)=k_{1}^{2} A_{1}^{-2} a(t) A^{-2}(t)$. And so on.

Remark 1 Let us take

$$
\begin{aligned}
& \alpha=\beta=1, \quad k A_{1}=c_{3} \sqrt{\frac{-k_{2} m^{2}}{k_{4}\left(2 m^{2}-1\right)}}, \\
& k_{2}=c_{2}, \quad k_{1}=c_{1} \sqrt{\frac{k_{2}}{\left(2 m^{2}-1\right)}}, \quad a(t) \rightarrow \beta(t), \quad b(t) \rightarrow \delta(t), \\
& c(t) \rightarrow \alpha(t), \quad t \rightarrow z, \quad x \rightarrow t .
\end{aligned}
$$


We find that $u_{1}$ turns to the solutions $u_{31}$ in Ref. [25],

$$
\begin{aligned}
u_{1_{1.1}}= & c_{3} \sqrt{\frac{-k_{2} m^{2}}{k_{4}\left(2 m^{2}-1\right)}} e^{\int_{0}^{z} \alpha(\tau) d \tau+i\left[c_{2} t+\frac{1}{2} \int_{0}^{z}\left(c_{1}^{2} k_{2}-c_{2}^{2}\right) \beta(\tau) d \tau\right]} \\
& \times c n\left[c_{1} \sqrt{\frac{k_{2}}{2 m^{2}-1}}\left(t-c_{2}\right) \int_{0}^{z} \beta(\tau) d \tau\right] .
\end{aligned}
$$

Let us take

$$
\begin{aligned}
& \alpha=\beta=1, \quad m=1, \quad k A_{1}=c R, \quad k_{2}=C_{1}, \\
& k_{1}=\sqrt{R} C_{2}, \quad a(t) \rightarrow \beta(t), \quad b(t) \rightarrow \delta(t), \quad c(t) \rightarrow \alpha(t), \\
& t \rightarrow z, \quad t \rightarrow x .
\end{aligned}
$$

We find that $u_{1_{1}}$ has degenerated to the famous bright-soliton solutions $u_{31}$ in Ref. [26].

$$
u_{1_{1.2}}=c R e^{\int_{0}^{z} \alpha(\tau) d \tau+i\left[C_{1} t+\frac{1}{2} \int_{0}^{z}\left(C_{2}^{2} R-C_{1}^{2}\right) \beta(\tau) d \tau\right]} \operatorname{sech}\left\{\sqrt{R}\left[C_{2} t-C_{1} C_{2} \int_{0}^{z} \beta(\tau) d \tau\right]\right\} .
$$

Let us take

$$
\begin{aligned}
& \alpha=\beta=1, \quad m=1, \quad k=\sqrt{-\frac{c_{2}}{2 c_{4}}}, \quad k_{2}=A_{3}, \quad k_{1}=\sqrt{\frac{2 c_{2}}{-m^{2}-1}} A_{2}, \\
& a(t) \rightarrow \beta(t), \quad b(t) \rightarrow \alpha(t), \\
& c(t) \rightarrow \gamma(t), \quad t \rightarrow z, \quad t \rightarrow x .
\end{aligned}
$$

We find that $u_{1_{2}}$ has degenerated to the famous dark-soliton solutions

$$
\begin{aligned}
u_{1_{2.1}}= & A_{1} \sqrt{-\frac{c_{2}}{2 c_{4}}} e^{\int_{0}^{z} \gamma(\tau) d \tau+i\left[A_{3} t+\frac{2 A_{2}^{2} c_{2}-A_{3}^{2}}{2} \int_{0}^{z} \beta(\tau) d \tau\right]} \\
& \times \tanh \left[A_{2} \sqrt{-c_{2}}\left(t-A_{3} \int_{0}^{z} \beta(\tau) d \tau\right)\right], \quad c_{2}<0, c_{4}>0 .
\end{aligned}
$$

Case 2

$$
\begin{aligned}
& A_{0}=\text { const }, \quad A_{1}=\frac{4 a_{4} A_{0}}{a_{3}}, \quad 8 a_{1} a_{4}^{2}=4 a_{2} a_{3} a_{4}-a_{3}^{3}, \quad a_{3} a_{4} \neq 0, \\
& c_{2}(t)=\left(\frac{a_{1} a_{4} k_{1}^{2}}{a_{3}}-\frac{a_{3}^{2} k_{1}^{2}}{16 a_{4}}-\frac{k_{2}^{2}}{2}\right) a(t), \quad b(t)=-\frac{a_{3}^{2} k_{1}^{2}}{16 a_{4} A_{0}^{2}} a(t) A^{-2}(t) .
\end{aligned}
$$

We acquire the following exact solutions of Eq. (2) by using Appendix B in Ref. [28]:

$$
\begin{aligned}
& u_{2_{j}}= k A_{0}\left(1+\frac{4 a_{4}}{a_{3}}\right) e^{\frac{\alpha}{\Gamma(1+\alpha)} \int_{0}^{t} \tau^{\alpha-1} c(\tau) d \tau+i\left[\frac{k_{2} x^{\beta}}{\Gamma(1+\beta)}+\frac{1}{\Gamma(\alpha)} \int_{0}^{t}(t-\tau)^{\alpha-1}\left(\frac{a_{1} a_{4} k_{1}^{2}}{a_{3}}-\frac{a_{3}^{2} k_{1}^{2}}{16 a_{4}}-\frac{k_{2}^{2}}{2}\right) a(\tau) d \tau\right]} \\
& \times F_{2_{j}}\left[\frac{k_{1} x^{\beta}}{\Gamma(1+\beta)}-\frac{k_{1} k_{2}}{\Gamma(\alpha)} \int_{0}^{t}(t-\tau)^{\alpha-1} a(\tau) d \tau\right] \\
& j=1,2,3, \ldots, 9 .
\end{aligned}
$$


If we let $a_{0_{4}}=1, a_{1_{4}}=-4, a_{2_{4}}=8-4 m^{2}, a_{3_{4}}=4-4 m^{2}, a_{4_{4}}=8 m^{2}-8, F_{4}=\frac{c n \xi}{c n \xi \pm s n \xi d n \xi}$, we get the general Jacobi elliptic functions solution.

$$
\begin{aligned}
& u_{2_{4}}=-7 k A_{0} e^{\frac{\alpha}{\Gamma(1+\alpha)} \int_{0}^{t} \tau^{\alpha-1} c(\tau) d \tau+i\left[\frac{k_{2} x^{\beta}}{\Gamma(1+\beta)}+\frac{1}{\Gamma(\alpha)} \int_{0}^{t}(t-\tau)^{\alpha-1}\left(8 k_{1}^{2}-\frac{\left(m^{2}-1\right) k_{1}^{2}}{8}-\frac{k_{2}^{2}}{2}\right) a(\tau) d \tau\right]} \frac{c n \xi}{c n \xi \pm s n \xi d n \xi}, \\
& \xi=\frac{k_{1} x^{\beta}}{\Gamma(1+\beta)}-\frac{k_{1} k_{2}}{\Gamma(\alpha)} \int_{0}^{t}(t-\tau)^{\alpha-1} a(\tau) d \tau .
\end{aligned}
$$

We have the consistency conditions $b(t)=-\frac{\left(m^{2}-1\right) k_{1}^{2}}{8 A_{0}^{2}} a(t) A^{-2}(t)$.

If we let $a_{0_{7}}=\frac{1}{4}, a_{17}=1, a_{27}=2-m^{2}, a_{37}=2-2 m^{2}, a_{47}=1-m^{2}, F_{7}=\frac{s n \xi}{1-\operatorname{sn} \xi+c n \xi}$, we have

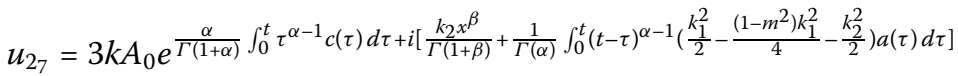

$$
\begin{aligned}
& \times\left(\operatorname{sn}\left[\frac{k_{1} x^{\beta}}{\Gamma(1+\beta)}-\frac{k_{1} k_{2}}{\Gamma(\alpha)} \int_{0}^{t}(t-\tau)^{\alpha-1} a(\tau) d \tau\right]\right) \\
& /\left(1-s n\left[\frac{k_{1} x^{\beta}}{\Gamma(1+\beta)}-\frac{k_{1} k_{2}}{\Gamma(\alpha)} \int_{0}^{t}(t-\tau)^{\alpha-1} a(\tau) d \tau\right]\right. \\
& \left.+c n\left[\frac{k_{1} x^{\beta}}{\Gamma(1+\beta)}-\frac{k_{1} k_{2}}{\Gamma(\alpha)} \int_{0}^{t}(t-\tau)^{\alpha-1} a(\tau) d \tau\right]\right),
\end{aligned}
$$

with the consistency conditions $b(t)=-\frac{\left(1-m^{2}\right) k_{1}^{2}}{4 A_{0}^{2}} a(t) A^{-2}(t)$. And so on.

Remark 2 The solutions $u_{1_{j}}$ and $u_{2_{j}}$ are new exact solutions for Eq. (2) to the best of our knowledge. When the modulus of these Jacobi elliptic functions solutions has degenerated to 1 or 0 , we can obtain the corresponding solitary-like solutions and triangular-like functions solutions. Some structure of these solutions of Eq. (1) are simulated in Fig. 1-Fig. 4

\section{The MFVIM and approximate solutions for the VFNLS}

According to the idea of FVIM [17, 20] and MFVIM [21], we can build a correction functional for Eq. (2) as follows:

$$
u_{n+1}=u_{n}+J_{t}^{\alpha}\left\{\lambda(t, x)\left[i \frac{\partial^{\alpha} u_{n}}{\partial t^{\alpha}}+\frac{1}{2} a(t) \frac{\partial^{2 \beta} \tilde{u}_{n}}{\partial x^{2 \beta}}+b(t) \tilde{u}_{n}\left|\tilde{u}_{n}\right|^{2}-i c(t) \tilde{u}_{n}\right]\right\}
$$
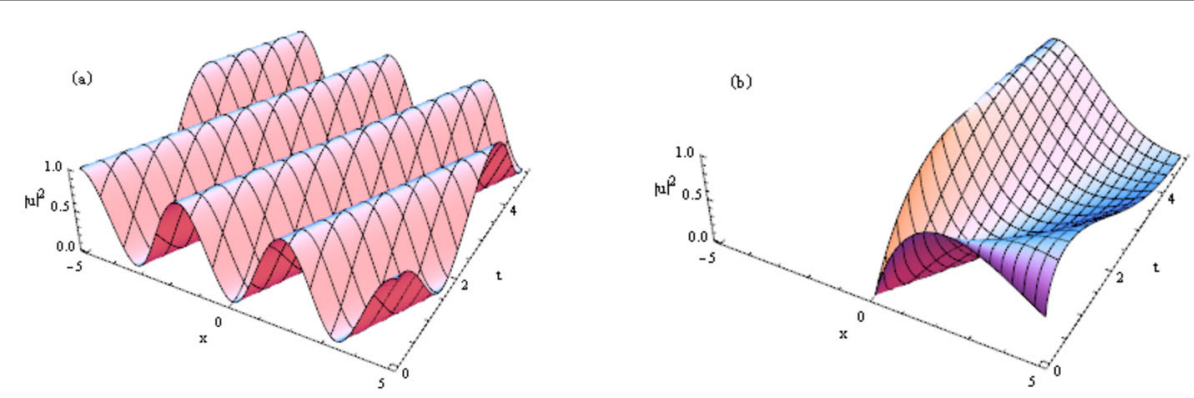

Figure 1 (a) The evolution plot of the exact doubly periodic solutions $u_{1}$, with the parameters $k=k_{1}=k_{2}=A_{1}=1, a(t)=1, c(t)=0, m=0.2, \alpha=\beta=1$. (b) $\alpha=0.5, \beta=0.5$ 

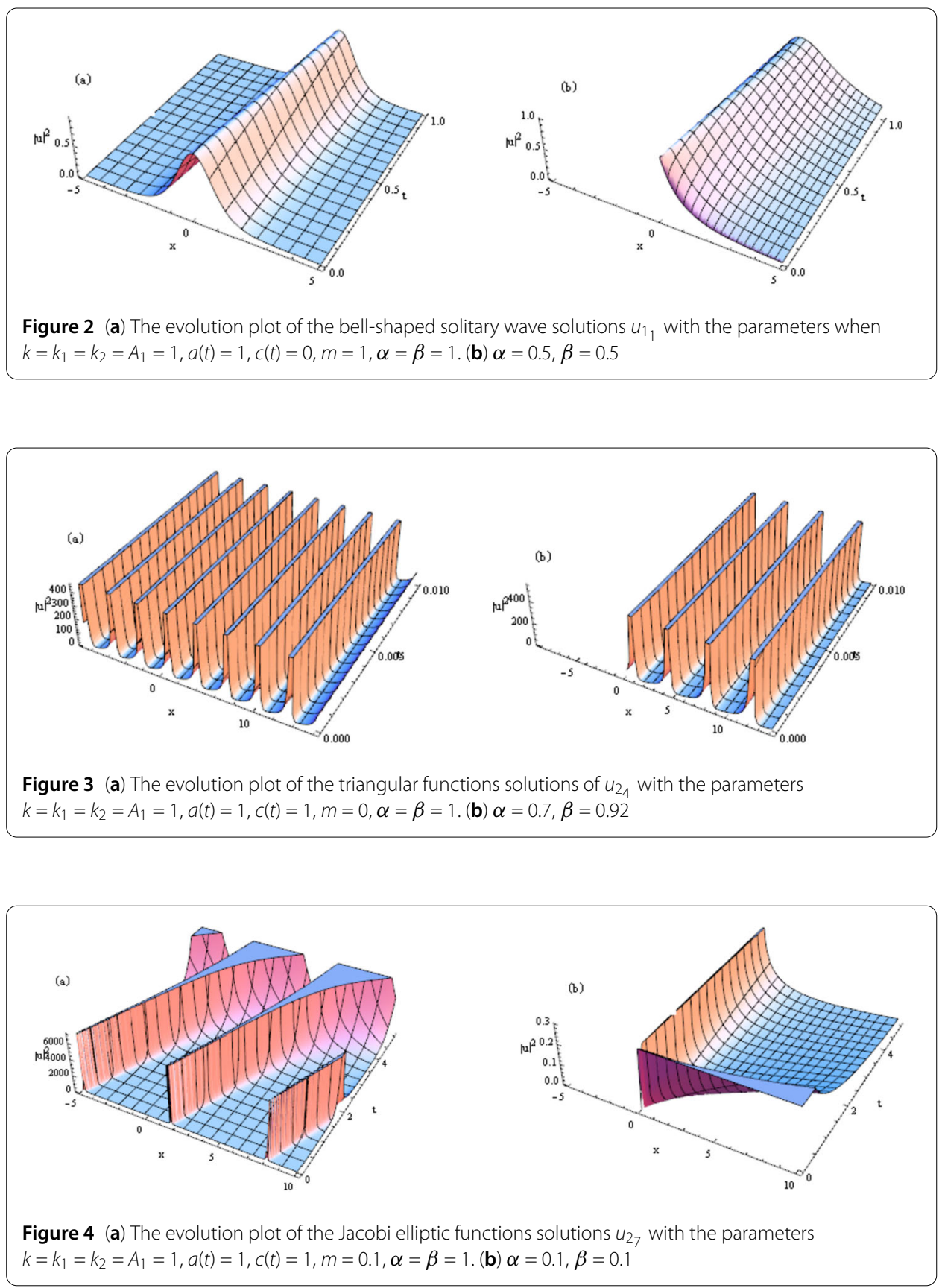

Here $u_{0}=u(x, 0)=f(x), \lambda(t, x)$ is a general Lagrange multiplier, which can be identified optimally with the variational theory. The function $\tilde{u}_{n}$ is a restricted variation, which means $\delta \tilde{u}_{n}=0$. Therefore, we first determine the Lagrange multiplier $\lambda(t, x)$ that will be identified optimally via integration by parts. The successive approximations $u_{n+1}, n \geq 0$, of the solution $u(x, t)$ will be readily obtained upon using the obtained $\lambda(t, x)$ and any selective function $u_{0}$. The initial values are usually used for choosing the zeroth approximation $u_{0}$. Consequently, the exact solution may be procured by using $u=\lim _{n \rightarrow \infty} u_{n}$.

In the following, we will apply the MFVIM to a model about Eq. (2) to illustrate the strength of the method. 
Example We now consider the following time-space fractional NLS equation [30-32]:

$$
i \frac{\partial^{\alpha} u}{\partial t^{\alpha}}+a \frac{\partial^{2 \beta} u}{\partial x^{2 \beta}}+b u|u|^{2}-i c u=0, \quad t>0,0<\alpha, \beta \leq 1, \quad u(x, 0)=A e^{i x}
$$

Here $a, b, c$ are constants. This equation occurs in various kinds of theoretical physics, such as nonlinear optics, superconductivity and plasma physics, which also can represent some dynamical system in quantum mechanics, fluid dynamics and nonlinear dynamics [31].

The correction functional for (10) reads

$$
u_{n+1}=u_{n}+J_{t}^{\alpha}\left\{\lambda(t, x)\left[i \frac{\partial^{\alpha} u_{n}}{\partial t^{\alpha}}+a \frac{\partial^{2} \tilde{u}_{n}}{\partial x^{2}}+b \tilde{u}_{n}\left|\tilde{u}_{n}\right|^{2}-i c \tilde{u}_{n}\right]\right\} .
$$

Making the above correction functional stationary,

$$
\delta u_{n+1}=\delta u_{n}+\lambda(t, x) i \delta u_{n}-J_{t}^{\alpha}\left\{\left[i \lambda^{\alpha}(t, x) \delta u_{n}\right]\right\} .
$$

After getting the coefficients of $\delta u_{n}$ to zero we can determine the Lagrange multiplier

$$
\lambda=i
$$

We produces the iteration formulation as follows:

$$
u_{n+1}=u_{n}+i J_{t}^{\alpha}\left[i \frac{\partial^{\alpha} u_{n}}{\partial t^{\alpha}}+a \frac{\partial^{2} u_{n}}{\partial x^{2}}+b u_{n}|u|^{2}-i c u_{n}\right], \quad|u|^{2}=\left|u_{0}\right|^{2} .
$$

As stated before, we can select $u_{0}=u(x, 0)=A e^{i x}$, using the iteration (14) and the Mathematica software, we obtain the following successive approximations:

$$
\begin{aligned}
u_{0}= & A e^{i x}, \\
u_{1}= & A e^{i x}+a A e^{i(x+\pi \beta)} \frac{i t^{\alpha}}{\Gamma(1+\alpha)}+b A^{3} e^{i x} \frac{i t^{\alpha}}{\Gamma(1+\alpha)}-i c A e^{i x} \frac{i t^{\alpha}}{\Gamma(1+\alpha)} \\
= & A e^{i x}\left(1+\frac{c_{1} i t^{\alpha}}{\Gamma(1+\alpha)}\right), \quad c_{1}=a e^{i \pi \beta}+b A^{2}-i c, \\
u_{2}= & u_{1}-A e^{i x} \frac{c_{1} i t^{\alpha}}{\Gamma(1+\alpha)}+a A e^{i(x+\pi \beta)}\left[\frac{i t^{\alpha}}{\Gamma(1+\alpha)}+\frac{c_{1} i^{2} t^{2 \alpha}}{\Gamma(1+2 \alpha)}\right] \\
& +b A^{3} e^{i x}\left[\frac{i t^{\alpha}}{\Gamma(1+\alpha)}+\frac{c_{1} i^{2} t^{2 \alpha}}{\Gamma(1+2 \alpha)}\right] \\
& -i c A e^{i x}\left[\frac{i t^{\alpha}}{\Gamma(1+\alpha)}+\frac{c_{1} i^{2} t^{2 \alpha}}{\Gamma(1+2 \alpha)}\right] \\
= & A e^{i x}\left[1+\frac{c_{1} i t^{\alpha}}{\Gamma(1+\alpha)}+\frac{c_{1}^{2} i^{2} t^{2 \alpha}}{\Gamma(1+2 \alpha)}\right], \\
u_{3}= & A e^{i x}\left[1+\frac{c_{1} i t^{\alpha}}{\Gamma(1+\alpha)}+\frac{c_{1}^{2} i^{2} t^{2 \alpha}}{\Gamma(1+2 \alpha)}+\frac{c_{1}^{3} i^{3} t^{3 \alpha}}{\Gamma(1+3 \alpha)}\right]
\end{aligned}
$$




$$
u_{n}=A e^{i x} \sum_{k=0}^{n} \frac{1}{\Gamma(1+k \alpha)}\left[c_{1} i t^{\alpha}\right]^{k} \text {. }
$$

The exact solution of Eq. (10) is

$$
u_{\text {exact }}=A e^{i x} \lim _{n \rightarrow \infty} \sum_{k=0}^{n} \frac{1}{\Gamma(1+k \alpha)}\left[c_{1} i t^{\alpha}\right]^{k}=A e^{i x} E_{\alpha}\left[\left(a e^{i \pi \beta}+b A^{2}-i c\right) i t^{\alpha}\right] .
$$

Here $E_{\alpha}\left[\left(a e^{i \pi \beta}+b A^{2}-i c\right) i t^{\alpha}\right.$ is the Mittag-Leffler function.

Remark 3 If we select $A=1, a=k, b=2 k, c=0, \alpha=1$, the solution (15) contains the result (49) in Ref. [30], the result (3.18) and (3.21) in Ref. [31], the result (27) in Ref. [32], but we can find that this iteration is much more simple, standard and powerful than the HAM, the ADM and the VIM mentioned in Refs. [30-32]. The solution (15) is a new exact solution for Eq. (10) to the best of our knowledge.

\section{Conclusion}

In this paper, the modified general mapping deformation method and the MFVIM are used for finding exact and approximate solutions of the GFNLS equation with the Caputo derivative. The obtained results indicate that the MFVIM is an effective, and a convenient and powerful method for solving nonlinear fractional complex differential equations when compared with some other traditional asymptotic decomposition method such as HAM, VIM and ADM. We believe that these two methods should play an important role for finding exact and approximate solutions in mathematical physics.

\section{Acknowledgements}

The authors wish to express their sincere appreciation to the editor and the anonymous referees for his (or her) valuable comments and suggestions.

\section{Funding}

This project was partially supported by the National Nature Science Foundation of China (Grant No. 61070231), Jiangsu university students practical innovation training program guidance project of Jiangsu Province (Grant No.

201811276060X, 201911276109H), Natural science research projects of Institutions of higher learning in Jiangsu Province (Grant No. 18KJB110013) and Nanjing Institute of Technology (Grant No. ZK201513, CKJB201709).

Availability of data and materials

The authors affirmed the availability of data and material in deriving the solutions mentioned in this manuscript.

\section{Competing interests}

The authors declare that they have no competing interests.

\section{Authors' contributions}

All authors contributed equally. All authors read and approved the final manuscript.

\section{Author details}

'Department of Mathematical Physics, Nanjing Institute of Technology, Nanjing, P.R. China. ${ }^{2}$ Faculty of Science, Jiangsu University, Zhenjiang, P.R. China. ${ }^{3}$ School of Electric Power Engineering, Nanjing Institute of Technology, Nanjing, P.R. China.

\section{Publisher's Note}

Springer Nature remains neutral with regard to jurisdictional claims in published maps and institutional affiliations. 


\section{References}

1. Zaslavsky, G.M.: Book review: Theory and applications of fractional differential equations by Anatoly A. Kilbas, Hari M. Srivastava and Juan J. Trujillo. Fractals 15(1), 101-102 (2011)

2. Tavazoei, M.S., Haeri, M., Jafari, S., et al.: Some applications of fractional calculus in suppression of chaotic oscillations. IEEE Trans. Ind. Electron. 55(11), 4094-4101 (2008)

3. Li, X.X., Tian, D., He, C.H., He, J.H.: A fractal modification of the surface coverage model for an electrochemical arsenic sensor. Electrochim. Acta 296, 491-493 (2019)

4. Machado, J.A.T., Silva, M.F., Barbosa, R.S., et al.: Some applications of fractional calculus in engineering. Math. Probl. Eng. 2010, 639801 (2010)

5. Esen, A., Sulaiman, T.A., Bulut, H., Baskonus, H.M.: Optical solitons to the space-time fractional $(1+1)$-dimensional coupled nonlinear Schrödinger equation. Optik 167, 150-156 (2018)

6. Zhang, Y., Liu, Y.P.: Darboux transformation and explicit solutions for $(2+1)$-dimensional nonlocal Schrödinger equation. Appl. Math. Lett. 92, 29-34 (2018)

7. Wang, J.J., Xiao, A.G.: Conservative Fourier spectral method and numerical investigation of space fractional Klein-Gordon-Schrödinger equations. Appl. Math. Comput. 350, 348-365 (2019)

8. Wu, G.C.: A fractional characteristic method for solving fractional partial differential equations. Appl. Math. Lett. 24, 1046-1050 (2011)

9. Zayed, E.M.E., Abdelaziz, M.A.M.: Applications of a generalized extended ( $\left.G^{\prime} / G\right)$-expansion method to find exact solutions of two nonlinear Schrödinger equations with variable coefficients. Acta Phys. Pol. A 121(3), 573-580 (2012)

10. Das, S., Vishal, K., Gupta, P.K., Yildirim, A.: An approximate analytical solution of time-fractional telegraph equation. Appl. Math. Comput. 217, 7405-7411 (2011)

11. Gepreel, K.A.: The homotopy perturbation method applied to the nonlinear fractional Kolmogorov-Petrovskii-Piskunov equations. Appl. Math. Lett. 24, 1428-1434 (2011)

12. Song, L.N., Wang, W.G.: A new improved Adomian decomposition method and its application to fractional differential equations. Appl. Math. Model. 37, 1590-1598 (2013)

13. He, J.H.: Variational iteration method - a kind of non-linear analytical technique: some examples. Int. J. Non-Linear Mech. 34, 699-708 (1999)

14. He, J.H.: An approximation to solution of space and time fractional telegraph equations by the variational iteration method. Math. Probl. Eng. 2012, 394212 (2012)

15. He, J.H.: A short remark on fractional variational iteration method. Phys. Lett. A 375, 3362-3364 (2011)

16. Noor, M.A., Mohyud-Din, S.T.: Variational iteration method for solving higher-order nonlinear boundary value problems using He's polynomials. Int. J. Nonlinear Sci. Numer. Simul. 9, 141-156 (2008)

17. Wang, Y., Deng, Q.G.: Fractal derivative model for tsunami travelling. Fractals 27, 1950017 (2019)

18. He, J.H.: Fractal calculus and its geometrical explanation. Results Phys. 10, 272-276 (2018)

19. Wang, K.L., Liu, S.Y.: He's fractional derivative for non-linear fractional heat transfer equation. Therm. Sci. 20(3), 793-796 (2016)

20. Anjum, N., He, J.H.: Laplace transform: making the variational iteration method easier. Appl. Math. Lett. 92, 134-138 (2019)

21. Hong, B.J., Lu, D.C.: Modified fractional variational iteration method for solving the generalized time-space fractional Schrödinger equation. Sci. World J. 2014, 964643 (2014)

22. Sakar, M.G., Erdogan, F., Yzldzrzm, A.: Variational iteration method for the time-fractional Fornberg-Whitham equation. Comput. Math. Appl. 63, 1382-1388 (2012)

23. Jumarie, G.: Table of some basic fractional calculus formulae derived from a modified Riemann-Liouville derivative for non-differentiable functions. Appl. Math. Lett. 22, 378-385 (2009)

24. Jumarie, G.: Derivation of an amplitude of information in the setting of a new family of fractional entropies. Inf. Sci. 216, 113-137 (2012)

25. Chen, Y., Li, B.: An extended subequation rational expansion method with symbolic computation and solutions of the nonlinear Schrödinger equation model. Nonlinear Anal. Hybrid Syst. 2, 242-255 (2008)

26. Li, B., Chen, Y.: On exact solutions of the nonlinear Schrödinger equations in optical fiber. Chaos Solitons Fractals 21 $241-247(2004)$

27. Lu, X., Zhu, H.W., Meng, X.H., Yang, Z.C., Tian, B.: Soliton solutions and a Bäcklund transformation for a generalized nonlinear Schrödinger equation with variable coefficients from optical fiber communications. J. Math. Anal. Appl. 336(2), 1305-1315 (2007)

28. Hong, B.J., Lu, D.C.: New exact solutions for the generalized variable-coefficient Gardner equation with forcing term Appl. Math. Comput. 219, 2732-2738 (2012)

29. Ebaid, A., Aly, E.H.: Exact solutions for the transformed reduced Ostrovsky equation via the F-expansion method in terms of Weierstrass-elliptic and Jacobian-elliptic functions. Wave Motion 49, 296-308 (2012)

30. Ganjiani, M.: Solution of nonlinear fractional differential equations using homotopy analysis method. Appl. Math Model. 34, 1634-1641 (2010)

31. Herzallahm, M.A.E., Gepreel, K.A.: Approximate solution to the time-space fractional cubic nonlinear Schrödinger equation. Appl. Math. Model. 36(11), 5678-5685 (2012)

32. Wazwaz, A.M.: A study on linear and nonlinear Schrödinger equations by the variational iteration method. Chaos Solitons Fractals 37, 1136-1142 (2008) 